\title{
Orbital Linearization of Smooth Completely Integrable Vector Fields
}

Nguyen Tien $Z U N G$ †‡

$\dagger$ School of Mathematics, Shanghai Jiao Tong University, 800 Dongchuan Road, Minhang District, Shanghai 200240, P.R. China

¥ Institut de Mathématiques de Toulouse, UMR5219 CNRS, Université Paul Sabatier, 118 route de Narbonne, 31062 Toulouse, France

E-mail: tienzung.nguyen@math.univ-toulouse.fr

Received July 04, 2017, in final form November 30, 2017; Published online December 12, 2017 https://doi.org/10.3842/SIGMA.2017.093

\begin{abstract}
The main purpose of this paper is to prove the smooth local orbital linearization theorem for smooth vector fields which admit a complete set of first integrals near a nondegenerate singular point. The main tools used in the proof of this theorem are the formal orbital linearization theorem for formal integrable vector fields, the blowing-up method, and the Sternberg-Chen isomorphism theorem for formally-equivalent smooth hyperbolic vector fields.
\end{abstract}

Key words: integrable system; normal form; linearization; nondegenerate singularity

2010 Mathematics Subject Classification: 37G05; 58K50; 37J35

\section{Introduction}

The main purpose of this paper is to show the following orbital linearization theorem for smooth $\left(C^{\infty}\right)$ vector fields which admit a complete set of first integrals near a nondegenerate singular point:

Theorem 1.1. Let $X$ be a smooth vector field in a neighborhood of $O=(0, \ldots, 0)$ in $\mathbb{R}^{n}$, which vanishes at $O$ and satisfies the following conditions:

i) (complete integrability): $X$ admits $n-1$ functionally independent smooth first integrals $F_{1}, \ldots, F_{n-1}$, i.e., $X\left(F_{1}\right)=\cdots=X\left(F_{n-1}\right)=0$ and $\mathrm{d} F_{1} \wedge \cdots \wedge \mathrm{d} F_{n-1} \neq 0$ almost everywhere;

ii) (nondegeneracy 1$)$ : the semisimple part of the linear part of $X$ at $O$ is non-zero, and the $\infty$ jets of $F_{1}, \ldots, F_{n-1}$ at $O$ are functionally independent (i.e., the $\infty$-jet of $\mathrm{d} F_{1} \wedge \cdots \wedge \mathrm{d} F_{n-1}$ at $O$ is non-zero);

iii) (nondegeneracy 2): If moreover 0 is an eigenvalue of $X$ at $O$ with multiplicity $k \geq 1$, then the differentials of the functions $F_{1}, \ldots, F_{k}$ are linearly independent at $O: \mathrm{d} F_{1}(O) \wedge \cdots \wedge$ $\mathrm{d} F_{k}(O) \neq 0$. Then there exists a local smooth coordinate system $\left(x_{1}, \ldots, x_{n}\right)$ in which $X$ can be written as

$$
X=F X^{(1)}
$$

where $X^{(1)}$ is a semisimple linear vector field in $\left(x_{1}, \ldots, x_{n}\right)$, and $F$ is a smooth first integral of $X^{(1)}$, i.e., $X^{(1)}(F)=0$, with $F(O)=1$. 
The above theorem is in fact more than mere orbital linearization: not only that $X$ is orbitally equivalent to its linear part $X^{(1)}$, but also the factor $F$ in the expression $X=F X^{(1)}$ in a normalized coordinate system is a first integral of $X$ and $X^{(1)}$. In [13], this kind of linearization is called geometric linearization.

The formal and analytic case of the above theorem also holds and was shown in [13] in a more general context of integrable non-Hamiltonian systems of type $(p, q)$, i.e., with $p$ commuting vector fields and $q$ common first integrals, where $p+q=n$ is the dimension of the manifold. The vector fields that we study in this paper are integrable of type $(1, n-1)$, i.e., just one vector field and $n-1$ first integrals.

The nondegeneracy condition in Theorem 1.1 is a bit stronger than the nondegeneracy condition in [13]: in [13] the (formal or analytic) vector field $X$ is called integrable nondegenerate if it satisfies the above conditions i) and ii), without the need of condition iii). (A priori, the condition that $\mathrm{d} F_{1} \wedge \cdots \wedge \mathrm{d} F_{n-1}(O) \neq 0$ is quite stronger than the condition that the $\infty$-jet of $\mathrm{d} F_{1} \wedge \cdots \wedge \mathrm{d} F_{n-1}$ at $O$ is not zero.) However, in fact, in the formal and analytic case, condition iii) is a simple consequence of the first two conditions and the theorem about the existence of (formal or analytic) Poincaré-Dulac normalization $[12,13]$. On the other hand, in the smooth case, we don't have a proof of the fact that condition iii) follows from conditions i) and ii) in general, though we do have a proof of this fact for dimension 2 .

The rest of this paper is organized as follows. Section 2 is devoted to some preliminary results, including the classification of nondegenerate singularities of completely integrable vector fields into (strong/weak) elliptic and hyperbolic cases (Lemma 2.2), and the normalization up to a flat term (Proposition 2.3). These preliminary results are used in the proof of Theorem 1.1 which is presented in Section 3. Finally, in Section 4, we show that, at least in the case $n=2$, condition iii) of Theorem 1.1 is a consequence of the first two conditions, and can be dropped from the formulation of the theorem (Theorem 4.1). We conjecture that condition iii) is redundant in the higher-dimensional case as well.

This paper is part of our program of systematic study of the geometry and topology of integrable non-Hamiltonian systems. In particular, Theorem 4.1, which is a refinement of Theorem 1.1 in the case of dimension 2, is the starting point of our joint work with Nguyen Van Minh on the local and global smooth invariants of integrable dynamical systems on 2-dimensional surfaces [15]. In connection with our results, we would like to mention the theorem of Chaperon on smooth equivalence of formally equivalent weakly hyperbolic systems [2], and its recent application to smooth geometric linearization of some classes of integrable non-Hamiltonian systems by Jiang [6]. We believe that Chaperon's techniques will be a key element in our smooth linearization problem, see also [14].

\section{Preliminary results}

\subsection{Adapted first integrals}

We have the following simple lemma, which is similar to the well-known Ziglin's lemma [11].

Lemma 2.1. Let $G_{1}, \ldots, G_{m}$ be $m$ formal series in $n$ variables which are functionally independent. Then there exist $m$ polynomial functions of $m$ variables $P_{1}, \ldots, P_{m}$ such that the homogeneous (i.e., lowest degree) parts of the formal series of $P_{1}\left(G_{1}, \ldots, G_{m}\right), \ldots, P_{m}\left(G_{1}, \ldots, G_{m}\right)$ are functionally independent.

The proof of the above lemma follows exactly the same lines as the proof of Ziglin of his lemma in [11], and our situation is simpler than the situation of meromorphic functions considered by Ziglin. 
Let $X$ be a smooth completely integrable vector field with a singularity at $O$. We will say that the smooth first integrals $F_{1}, \ldots, F_{n-1}$ of $X$ are adapted first integrals if

$$
\mathrm{d} H_{1} \wedge \cdots \wedge \mathrm{d} H_{n-1} \neq 0 \quad \text { a.e. }
$$

where $H_{i}=F_{i}^{\left(h_{i}\right)}$ denotes the homogeneous part (consisting of non-constant terms of lowest degree in the Taylor expansion) of $F_{i}$ at $O$. Using the above lemma to replace the first integrals $F_{1}, \ldots, F_{n-1}$ of $X$ by appropriate polynomial functions of them if necessary, from now on we can assume that $F_{1}, \ldots, F_{n-1}$ are adapted.

\subsection{The eigenvalues of $X$}

The fact that $X$ admits $n-1$ first integrals implies that $X$ is very resonant at $O$. More precisely, we have:

Lemma 2.2. Let $\left(X, F_{1}, \ldots, F_{n-1}\right)$ be smooth nondegenerate at $O$, i.e., they satisfy the conditions of Theorem 1.1. Then the linear part of $X$ at $O$ is semisimple, and there is a positive number $\lambda>0$ such that either all the eigenvalues of $X$ at $O$ belong to $\lambda \mathbb{Z}$, or all of them belong to $\sqrt{-1} \lambda \mathbb{Z}$.

Proof. We can assume that $H_{1}, \ldots, H_{n-1}$ are functionally independent, where $H_{i}$ denotes the homogeneous part of $F_{i}$. The equality $X\left(F_{i}\right)=0$ implies that

$$
X^{s s}\left(H_{i}\right)=X^{(1)}\left(H_{i}\right)=0 \quad \forall i=1, \ldots, n-1,
$$

where $X^{(1)}$ is the linear part of $X$, and $X^{s s}$ is the semisimple part of $X^{(1)}$ in the Jordan decomposition (see, e.g., $[12,13])$. We can write

$$
X^{s s}=\sum_{i=1}^{n} \lambda_{i} z_{i} \frac{\partial}{\partial z_{i}}
$$

in a complex coordinate system. Recall that the ring of polynomial first integrals of $\sum_{i=1}^{n} \lambda_{i} z_{i} \frac{\partial}{\partial z_{i}}$ is generated by the monomial functions $\prod_{i=1}^{n} z_{i}^{a_{i}}$, which satisfy the resonance relation

$$
\sum_{i=1}^{n} a_{i} \lambda_{i}=0 .
$$

The fact that $H_{1}, \ldots, H_{n-1}$ are independent implies that equation (2.1) has $n-1$ linearly independent solutions which belong to $\mathbb{Z}_{+}^{n}$, which in turn implies that there is a complex number $\lambda$ such that $\lambda_{1}, \ldots, \lambda_{n} \in \lambda \mathbb{Z}$. Remark that if the spectrum of $X^{s s}$ contains a complex eigenvalue $\lambda_{1} \in \mathbb{C} \backslash(\mathbb{R} \cup \sqrt{-1} \mathbb{R})$, then its complex conjugate $\bar{\lambda}_{1}$ is also in the spectrum because $X$ is real, and $\lambda_{1}$ and $\bar{\lambda}_{1}$ cannot belong to $\lambda \mathbb{Z}$ at the same time for any $\lambda$. Thus any eigenvalue of $X^{s s}$ is either real or pure imaginary. If there is one real non-zero eigenvalue, then we can choose $\lambda \in \mathbb{R}_{+}$, otherwise we can choose $\lambda \in \sqrt{-1} \mathbb{R}_{+}$. Notice that $\lambda \neq 0$ because at least one eigenvalue of $X^{s s}$ is non-zero by our assumptions.

The common level sets of $H_{1}, \ldots, H_{n-1}$ are 1-dimensional almost everywhere, and since both $X^{(1)}$ and $X^{s s}$ are tangent to these common level sets, we have that $X^{(1)} \wedge X^{s s}=0$, which implies that $X^{(1)}$ is semisimple, i.e., $X^{(1)}=X^{s s}$.

With the above lemma, we can divide the problem into 4 cases (here $\mathbb{R}^{*}=\mathbb{R} \backslash\{0\}$ ): 
I. Strongly hyperbolic (or hyperbolic without eigenvalue 0 ): $\lambda_{i} \in \lambda \mathbb{R}^{*} \forall i$.

II. Weakly hyperbolic (or hyperbolic with eigenvalue 0): $\lambda_{i} \in \lambda \mathbb{R}^{*} \forall i>k \geq 1, \lambda_{1}=\cdots=$ $\lambda_{k}=0$.

III. Strongly elliptic (or elliptic without eigenvalue 0): $\lambda_{i} \in \sqrt{-1} \lambda \mathbb{R}^{*} \forall i$.

IV. Weakly elliptic (or elliptic with eigenvalue 0): $\lambda_{i} \in \sqrt{-1} \lambda \mathbb{R}^{*} \forall i>k \geq 1, \lambda_{1}=\cdots=\lambda_{k}$ $=0$.

\subsection{Linearization up to a flat term}

Using the geometric linearization theorem of [13] in the formal case, we get the following proposition:

Proposition 2.3 (linearization up to a flat term). Assume that $X$ satisfies the hypotheses of Theorem 1.1. Then there is a local smooth coordinate system $\left(x_{1}, \ldots, x_{n}\right)$ in which $X$ can be written as

$$
X=F X^{(1)}+\text { flat }
$$

where $X^{(1)}$ is the linear part of $X$ in the coordinate system $\left(x_{1}, \ldots, x_{n}\right), F$ is a smooth first integral of $X^{(1)}$, and flat means a smooth term which is flat at $O$.

Proof. Denote by $\hat{X}$ (resp. $\hat{F}_{i}$ ) the $\infty$-jet of $X$ (resp. $F_{i}$ ) at $O: \hat{X}$ is a formal vector field (resp. function) at $O$. If $\left(X, F_{1}, \ldots, F_{n-1}\right)$ is smooth nondegenerate at $O$, then $\left(\hat{X}, \hat{F}_{1}, \ldots, \hat{F}_{n-1}\right)$ is a nondegenerate formal integrable system of type $(1, n-1)$ at $p$. According to the geometric linearization theorem of [13], this formal integrable system can be linearized geometrically, i.e., there is a formal coordinate system $\left(\hat{x}_{1}, \ldots, \hat{x}_{n}\right)$ in which we have

$$
\hat{X}=\hat{F} \hat{X}^{(1)},
$$

where $\hat{X}^{(1)}$ is the linear part of $\hat{X}$ in the formal coordinate system $\left(\hat{x}_{1}, \ldots, \hat{x}_{n}\right)$, and $\hat{F}$ is a formal first integral of $\hat{X}^{(1)}$. By the classical Hilbert-Weyl theorem (see, e.g., Theorem 4.2 of Chapter XII of [5]) applied to the torus action associated to $X^{(1)}$ (see [12, 13] for this associated torus action), we can write

$$
\hat{F}=\hat{f}\left(Q_{1}\left(\hat{x}_{1}, \ldots, \hat{x}_{n}\right), \ldots, Q_{m}\left(\hat{x}_{1}, \ldots, \hat{x}_{n}\right)\right),
$$

where $\hat{f}$ is a formal series and $Q_{1}\left(\hat{x}_{1}, \ldots, \hat{x}_{n}\right), \ldots, Q_{m}\left(\hat{x}_{1}, \ldots, \hat{x}_{n}\right)$ are homogeneous polynomials generating the ring of polynomial first integrals of $\hat{X}^{(1)}$. Using Borel theorem, we get a smooth coordinate system $\left(x_{1}, \ldots, x_{n}\right)$ whose $\infty$-jet is $\left(\hat{x}_{1}, \ldots, \hat{x}_{n}\right)$, and a smooth function $f$ of $m$ variables whose $\infty$-jet is $\hat{f}$. Put

$$
F\left(x_{1}, \ldots, x_{n}\right)=f\left(Q_{1}\left(x_{1}, \ldots, x_{n}\right), \ldots, Q_{m}\left(x_{1}, \ldots, x_{n}\right)\right) .
$$

Then equations (2.2), (2.3) and (2.4) imply that $X=F X^{(1)}+$ flat in the smooth coordinate system $\left(x_{1}, \ldots, x_{n}\right)$.

\subsection{Reduction to the case without eigenvalue 0}

Assume that $X$ has zero eigenvalue at $O$ with multiplicity $k$, and $\mathrm{d} F_{1} \wedge \cdots \wedge \mathrm{d} F_{k}=0$, i.e., we can use $F_{1}, \ldots, F_{k}$ as the first $k$ coordinates in our local coordinate systems. Since the vector field $X$ preserves $x_{1}, \ldots, x_{k}$, we can view it as a $k$-dimensional family of vector fields on $(n-k)$-dimensional spaces

$$
U_{c_{1}, \ldots, c_{k}}=\left\{F_{1}=c_{1}, \ldots, F_{k}=c_{k}\right\}
$$


(for $c_{1}, \ldots, c_{k}$ small enough). It follows from the usual implicit function theorem and the nondegeneracy condition that on each $U_{c_{1}, \ldots, c_{k}}$ there is a unique point $O_{c_{1}, \ldots, c_{k}}$ such that $X\left(O_{c_{1}, \ldots, c_{k}}\right)$ $=0$, and moreover the point $O_{c_{1}, \ldots, c_{k}}$ depends smoothly on $c_{1}, \ldots, c_{k}$, the eigenvalues of $X$ at $c_{1}, \ldots, c_{k}$, are non-zero. It also follows from the formal independence of $F_{1}, \ldots, F_{n}$ at $O$, that the functions $F_{k+1}, \ldots, F_{n}$ are formally independent at every point $O_{c_{1}, \ldots, c_{k}}$ provided that $c_{1}, \ldots, c_{k}$ are sufficiently small. In other words, we have a $k$-dimensional family of nondegenerate singularities of smooth completely integrable $(n-k)$-dimensional vector fields $X_{c_{1}, \ldots, c_{k}}$. In order to normalize $X$, it suffices to normalize $X_{c_{1}, \ldots, c_{k}}$ in a way which depends smoothly on the parameter.

\section{Proof of Theorem 1.1}

We will always assume that the vector field $X$ satisfies the hypotheses of Theorem 1.1. The fact that the linear part of $X$ is semisimple is established by Lemma 2.2. In view of Section 2.4, it suffices to prove Theorem 1.1 for the cases without zero eigenvalue, by a proof whose parametrized version also works the same.

\subsection{The hyperbolic case}

Assume that $X$ is hyperbolic without eigenvalue 0. According to Proposition 2.3, we can write $X=Y+$ flat, where $Y=F X^{(1)}$ is a smooth hyperbolic integrable vector field in normal form. Since $X$ and $Y$ are hyperbolic and coincide up to a flat term, Sternberg-Chen theorem [3, 10] says that $X$ is locally smoothly isomorphic to $Y$, i.e., there is a smooth coordinate system in which $X$ can be written as $X=F X^{(1)}$, where $F$ is a smooth first integral of $X^{(1)}$. Theorem 1.1 is proved in the hyperbolic case without eigenvalue 0 . This is also a special case of a result of Kai Jiang [6] on smooth linearization of weakly hyperbolic integrable vector fields.

\subsection{The elliptic case}

In this subsection, we will assume that all the eigenvalues of $X$ at $O$ are non-zero pure imaginary. Using Proposition 2.3, we can assume that $X=F X^{(1)}+$ flat in a local smooth coordinate system $\left(x_{1}, \ldots, x_{n}\right)$, where $F$ is a smooth function such that $F(O)=1$. Put $Y=X / F$. Then $Y$ has the same first integrals as $X$, and

$$
Y=X^{(1)}+\text { flat. }
$$

The fact that $X$ is of strong elliptic type implies immediately that the dimension $n$ is even, the eigenvalues of $X$ at $O$ are $\pm \sqrt{-1} a_{1}, \ldots, \pm \sqrt{-1} a_{n / 2}$ where $a_{1}, \ldots, a_{n / 2}$ are positive real numbers, and we can choose the coordinates $\left(x_{1}, \ldots, x_{n}\right)$ such that

$$
X^{(1)}=\sum_{i=1}^{n / 2} a_{i}\left(x_{2 i-1} \frac{\partial}{\partial x_{2 i}}-x_{2 i} \frac{\partial}{\partial x_{2 i-1}}\right) .
$$

According to Lemma 2.2, we can choose $\lambda>0$ such that $a_{1} / \lambda, \ldots, a_{n / 2} / \lambda$ are natural numbers whose greatest common divisor is 1 .

Lemma 3.1. Locally near $O$ all the orbits of $Y=X / F$ (except the fixed point $O$ ) are periodic, with periods which are uniformly bounded above and below.

Proof. The vector field $\left.\left(\mathrm{d} F_{1} \wedge \cdots \wedge \mathrm{d} F_{n-1}\right)\right\lrcorner\left(\frac{\partial}{\partial x_{1}} \wedge \cdots \wedge \frac{\partial}{\partial x_{n}}\right)$ is tangent to $Y$, and therefore it is divisible by $Y$ (by de Rham division theorem, because of the nondegeneracy condition, see, e.g., Appendix A.2 of [4]; in that appendix the de Rham theorem is given for differential forms, 
but it works the same for vector fields, via an isomorphism between the tangent bundle and the contangent bundle over a manifold), i.e., we can write

$$
\left.\left(\mathrm{d} F_{1} \wedge \cdots \wedge \mathrm{d} F_{n-1}\right)\right\lrcorner\left(\frac{\partial}{\partial x_{1}} \wedge \cdots \wedge \frac{\partial}{\partial x_{n}}\right)=G Y
$$

where $G$ is a smoth non-flat function at $O$. Notice that the singular locus of the map $\left(F_{1}, \ldots\right.$, $\left.F_{n-1}\right): U \rightarrow \mathbb{R}^{n-1}$, where $U \ni O$ is a small neighborhood of $O$ in $\mathbb{R}^{n}$, coincides with the zero locus of $G$.

It is clear that, by continuity, the set of all points $x \in U$ such that the orbit of $Y$ through $x$ is periodic of period $\leq 3 \pi / \lambda$ is a closed subset of $U$. We want to show that this set is actually equal to $U$ (provided that $U$ is small enough). Consider the singular locus

$$
S=\{x \in U \mid G(x)=0\}=\left\{x \in U \mid \mathrm{d} F_{1} \wedge \cdots \wedge \mathrm{d} F_{n-1}(x)=0\right\} .
$$

Since $G$ is non-flat at $O$, we can choose a coordinate system $\left(z_{1}, \ldots, z_{n}\right)$ which is a linear transformation of the coordinate system $\left(x_{1}, \ldots, x_{n}\right)$, such that the homogeneous part $G^{(h)}$ of $G$ has the form

$$
G^{(h)}=z_{1}^{h}+\cdots
$$

(where $h$ denotes the degree of $G^{(h)}$ ), which implies that $\frac{\partial^{h} G}{\partial z_{1}^{h}} \neq 0$ in $U$.

Because $\frac{\partial^{h} G}{\partial z_{1}^{h}}$ does not vanish in $U$, by the classical Rolle's theorem on each line $\left\{z_{2}=\right.$ const, $\ldots, z_{n}=$ const $\}$ in $U$ there are at most $h$ zeros of the function $G$, the intersection of the singular locus $S$ with each line $\left\{z_{2}=\right.$ const, $\ldots, z_{n}=$ const $\}$ in $U$ consists of at most $n-1$ points, and the function $G$ is not flat at any point of $S$.

Due to the ellipticity of the vector field $X^{(1)}$, there must be at least one index $j \neq 1$ such that the coefficient of the monomial term $z_{1} \frac{\partial}{\partial z_{j}}$ in $X^{(1)}$ in the coordinate system $\left(z_{1}, \ldots, z_{n}\right)$ is not zero. Without loss of generality, we may assume that the coefficient of the monomial term $z_{1} \frac{\partial}{\partial z_{n}}$ in $X^{(1)}$ is not zero.

Consider the local hyperplane

$$
P=\left\{z_{n}=0\right\} \subset U
$$

with the coordinate system $\left(z_{1}, \ldots, z_{n-1}\right)$. For each $\epsilon>0$ sufficiently small, denote by

$$
q_{\epsilon}=\left(z_{1}=\epsilon, z_{2}=0, \ldots, z_{n}=0\right) \in P
$$

the point in $U$ whose coordinate $z_{1}$ is equal to $\epsilon$ and the other coordinates $z_{i}$ vanish for every $i \geq 2$. Then the vector fields $X^{(1)}$ and $Y$ are transversal to $P$ at every point $q_{\epsilon}$ such that $\epsilon>0$ is sufficiently small.

Recall from Section 2.1 that we can, and will, assume that $F_{1}, \ldots, F_{n}$ to be adapted first integrals, i.e., the homogeneous part of $\mathrm{d} F_{1} \wedge \cdots \wedge \mathrm{d} F_{n}$ is equal to $\mathrm{d} H_{1} \wedge \cdots \wedge \mathrm{d} H_{n}$, where $H_{i}=F_{i}^{\left(h_{i}\right)}$ is the homogeneous part of $F_{i}$ for each $i=1, \ldots, n-1$ and $\operatorname{deg} H_{i}=h_{i}$. In particular, we have $h+1=\sum_{i=1}^{n-1}\left(h_{i}-1\right)$, where $h$ is the degree of the homogeneous part $G^{(h)}$ of the function $G$ in formulas (3.2) and (3.3), and $h+2 \geq h_{i}$ for any $i=1, \ldots, n-1$.

Denote by $B^{n-1}\left(q_{\epsilon}, \epsilon^{h+2}\right)$ the $(n-1)$-dimensional ball of center $q_{\epsilon}$ and radius $\epsilon^{h+2}$ on the local hyperplane $P=\left\{z_{n}=0\right\}$. We observe that, for every $\epsilon$ sufficiently small, the restriction of the map $\left(F_{1}, \ldots, F_{n-1}\right)$ to $B^{n-1}\left(q_{\epsilon}, \epsilon^{h+2}\right)$ is an injective map from $B^{n-1}\left(q_{\epsilon}, \epsilon^{h+2}\right)$ to $\mathbb{R}^{n-1}$.

Indeed, consider any two distinct points $\mathbf{p}, \mathbf{q} \in B^{n-1}\left(q_{\epsilon}, \epsilon^{h+2}\right), \mathbf{p} \neq \mathbf{q}$. Consider the constant unit vector field $Z_{\mathbf{p}, \mathbf{q}}=\frac{\mathbf{q}-\mathbf{p}}{\|\mathbf{q}-\mathbf{p}\|}$ on $U$ with respect to the coordinate system $\left(z_{1}, \ldots, z_{n}\right)$ which 
maps $U$ onto a neighborhood of the origin in the Euclidean space $\mathbb{R}^{n}$. The difference $\mathbf{q}-\mathbf{p}$ and the norm $\|\mathbf{q}-\mathbf{p}\|$ (i.e., the distance from $\mathbf{p}$ to $\mathbf{q}$ ) are taken with respect to this Euclidean structure. Due to the fact that our first integrals are adapted, at least one of the functions $Z_{\mathbf{p}, \mathbf{q}}\left(F_{1}\right), \ldots, Z_{\mathbf{p}, \mathbf{q}}\left(F_{n-1}\right)$, say $Z_{\mathbf{p}, \mathbf{q}}\left(F_{k}\right)$, has a homogeneous part of the type

$$
c_{\mathbf{p}, \mathbf{q}} z_{1}^{h_{k}-1}+\cdots,
$$

with a coefficient $c_{\mathbf{p}, \mathbf{q}} \neq 0$, and $k$ can be chosen as a function of $\mathbf{p}$ and $\mathbf{q}$ such that $\left|c_{(\mathbf{p}, \mathbf{q})}\right|$ is uniformly bounded by positive constants. Using standard division techniques (see, e.g., the Malgrange's preparation theorem [8], but the situation here is much simpler than this general theorem), one can decompose $Z_{\mathbf{p}, \mathbf{q}}\left(F_{k}\right)$ as

$$
Z_{\mathbf{p}, \mathbf{q}}\left(F_{k}\right)=f_{1, \mathbf{p}, \mathbf{q}} z_{1}^{h_{k}-1}+\sum_{i=2}^{n} z_{i} f_{i, \mathbf{p}, \mathbf{q}},
$$

where $f_{i, \mathbf{p}, \mathbf{q}}$ are smooth functions on $U$ depending uniformly continuously on the unit vector $Z_{\mathbf{p}, \mathbf{q}}$ (as long as the index $k$ remains the same), and with $f_{1, \mathbf{p}, \mathbf{q}}(0)=c_{\mathbf{p}, \mathbf{q}}$. For the points on $B^{n-1}\left(q_{\epsilon}, \epsilon^{h+2}\right)$ we have that $\epsilon-\epsilon^{h+2} \leq z_{1} \leq \epsilon+\epsilon^{h+2}$ while $\left|z_{i}\right| \leq \epsilon^{h+2} \leq \epsilon^{h_{k}}$ for all $i \geq 2$, hence in the above expression of $Z_{\mathbf{p}, \mathbf{q}}\left(F_{k}\right)$ the terms $z_{i} f_{i, \mathbf{p}, \mathbf{q}}$ with $i \geq 2$ are very small compared to the term $f_{1, \mathbf{p}, \mathbf{q}} z_{1}^{h_{k}-1}$, and so in particular the sign of $Z_{\mathbf{p}, \mathbf{q}}\left(F_{k}\right)$ does not change on $B^{n-1}\left(q_{\epsilon}, \epsilon^{h+2}\right)$ for $\epsilon$ sufficiently small, which implies that $F_{k}(\mathbf{q})-F_{k}(\mathbf{p}) \neq 0$ by the mean value theorem. Thus, we have shown the injectivity of $\left(F_{1}, \ldots, F_{n-1}\right)$ on $B^{n-1}\left(q_{\epsilon}, \epsilon^{h+2}\right)$ for every $\epsilon$ sufficiently small.

Consider now the Poincaré map (i.e., the first return map), denoted by $\phi$, defined on the ball $B^{n-1}\left(q_{\epsilon}, \epsilon^{h+2}\right) \subset P$ of the flow of $Y$ in $U$ (a priori the image of this map may lie a bit outside of $B^{n-1}\left(q_{\epsilon}, \epsilon^{h+2}\right)$ but on the same local hyperplane $\left.P\right)$. A priori this map does not necessarily fix the point $q_{\epsilon}$. But due to the fact the flow of $X^{(1)}$ is periodic (in particular, the Poincaré map for $X^{(1)}$ is the identity map) and the fact that $Y=X^{(1)}+$ flat (which implies that the Poincaré map $\phi$ for $Y$ deviates from the Poincaré map for $X$ by a flat term), we have that the distance from $q_{\epsilon}$ to $\phi\left(q_{\epsilon}\right)$ is a flat function in $\epsilon$. In particular, for every $\epsilon$ sufficiently small we have

$$
\mathrm{d}\left(q_{\epsilon}, \phi\left(q_{\epsilon}\right)\right)<\epsilon^{h+2}
$$

where $d$ denotes the Euclidean distance in the coordinate system $\left(z_{1}, \ldots, z_{n}\right)$, and hence the point $\phi\left(q_{\epsilon}\right)$ lies in the $(n-1)$-dimensional ball $B^{n-1}\left(q_{\epsilon}, \epsilon^{h+2}\right)$.

Due to the invariance of the functions $F_{i}$ with respect to the vector field $Y$, and hence with respect to the Poincaré map $\phi$, we also have that the points $q_{\epsilon}$ and $\phi\left(q_{\epsilon}\right)$ have the same image under the map $\left(F_{1}, \ldots, F_{n-1}\right)$. But this map is injective on the ball $B^{n-1}\left(q_{\epsilon}, \epsilon^{h+2}\right)$ which contains these two points, so in fact these two points must coincide, i.e., we have $q_{\epsilon}=\phi\left(q_{\epsilon}\right)$, and the orbit of the flow of $Y$ through the point $q_{\epsilon}$ is a periodic orbit, and the period of this orbit is equal to $2 \pi / \lambda$ plus a small error term which tends to 0 faster than any power of $\epsilon$ when $\epsilon$ tends to 0 .

Denote by $V$ the path-connected component of $U \backslash S$ which contains the points $q_{\epsilon}$. ( $V$ is not equal $U \backslash S$ in general). Then the orbit of $Y$ through any point $q \in V$ is also periodic and its period is close to $2 \pi / \lambda$ (the difference between the period and $2 \pi / \lambda$ tends to 0 uniformly when the radius of $U$ tends to 0 ). This fact can be proved easily by showing that the set of points of $V$ which satisfies the mentioned property is closed and open in $V$ at the same time: closed due to the continuity, and open because $\left(F_{1}, \ldots, F_{n-1}\right)$ is regular in $V$ and is preserved by the flow of $Y$.

Let $q \in S$ be a point in the locus $S$ which also lies on the boundary of $V$. Then by continuity, there is also a number $T$ near $2 \pi / \lambda$ such that the time- $T$ flow of $Y$ fixes the point $q$. In other words, the orbit of $Y$ through $q$ is also periodic, and the period is equal to $T$ or a fraction $T / m$ 
of $T$ for some natural number $m$. As before, consider a $(n-1)$-dimensional ball $B^{n-1}(q, \delta)$ which is centered at $q$ and orthogonal to $Y(q)$, for some $\delta>0$ small enough. Consider the Poincaré map $\phi$ of $Y$ on $B^{n-1}(q, \delta)$ corresponding to the time $T$ (i.e., if the period of the orbit through $q$ is $T / m$ then consider the $m$-time iteration of the usual Poincaré map). Since the intersection of $B^{n-1}(q, \delta)$ with $V$ contains an open subset of $B^{n-1}(q, \delta)$ whose closure contains $q$, and the Poincaré map is identity on that open subset by the above considerations, the Poincaré map on $B^{n-1}(q, \delta)$ is equal to the identity map plus a flat term at $q$. On the other hand, this Poincaré map must preserve the map $\left.\left(F_{1}, \ldots, F_{n-1}\right)\right|_{B^{n-1}(q, \delta)}$, and the determinant of the differential of this map is not flat at $q$. It implies that the Poincaré map must be identity in a small neighborhood of $q$ in $B^{n-1}(q, \delta)$. Thus, we can "engulf" the set of points shown to have periodic orbits from $V$ to a larger open subset of $U$ which contains the boundary of $V$. Continuing this engulfing process, we get that the set of points in $U$ having periodic orbits is actually the whole $U$.

We will linearize $Y=X / F$ orbitally, and then deduce the normalization of $X$ from this linearization. In order to do that, let us consider the blow-up of $\mathbb{R}^{n}$ at $O$, which will be denoted by

$$
p: E \rightarrow U
$$

where $U \ni O$ is a neighborhood of $O$ in $\mathbb{R}^{n}$ and $p^{-1}(O) \cong \mathbb{R} \mathbb{P}^{n-1}$ is the exceptional divisor of the blow-up in $E$. We will need the following simple lemma, whose proof is straightforward:

Lemma 3.2. With the above notations, a function $G$ or a vector field $Z$ is flat at $O$ in $U$ if and only if its pull-back to $E$ via the projection map $p$ is flat along $p^{-1}(O)$ in $E$.

(In the above lemma, $G$ and $Z$ are arbitrary, and the pull back from $U$ to $E$ means the continuous extention from $E \backslash p^{-1}(O)$ to $E$ of the pull-back by the diffeomorphism $p: E \backslash p^{-1}(O)$ $\rightarrow U \backslash\{O\}$, if such a continuous extension exists.)

Denote by $\tilde{G}$ (resp. $\tilde{Z}$ ) the pull-back of a function $G$ (resp. vector field $Z$ ) via the projection map $\pi: E \rightarrow U$ of the blow-up. Then we have

$$
\tilde{Y}=\tilde{X}^{(1)}+\tilde{Z}
$$

in $E$, where $\tilde{Z}$ is vector field which is flat along $p^{-1}(O)$, and $\tilde{X}^{(1)}$ is a smooth periodic vector field in $E$ of period $2 \pi / \lambda$. By Lemma 3.1, the orbits of $\tilde{Y}$ are closed, with periods close to the period of $\tilde{X}^{(1)}$. Due to the flatness of $Z$ along $p^{-1}(O)$, the period of $\tilde{Y}$ at the points in $E$ is equal to $2 \pi / \lambda$ plus a smooth function on $E$ which is flat along $p^{-1}(O)$. Projecting $\tilde{Y}$ back to $U$ and using Lemma 3.2, we get a smooth period function $P=2 \pi / \lambda+$ flat (which is invariant on the orbits) such that $P Y$ is periodic of period 1. In other words, $P Y$ generates a smooth $\mathbb{T}^{1}$-action. Using the classical Cartan-Bochner smooth linearization theorem for compact group actions, we find a smooth coordinate system, which we will denote again by $\left(x_{1}, \ldots, x_{n}\right)$, in which $P X / F=P Y$ is a linear vector field, i.e., in which we have

$$
X=G X^{(1)},
$$

where $G$ is a smooth function and $X^{(1)}$ is a linear vector field which satisfies formula (3.1).

A priori, the function $F$ given by Proposition 2.3 is not a first integral of $X$ (though it is a first integral of the linear part of $X$ in some coordinate system), and so the function $G=2 \pi F / P \lambda$ in formula (3.4) is not a first integral of $X$ either. But we can normalize further in order to change $G$ into a first integral. Indeed, by the arguments presented above, we can assume that $G$ is a smooth first integral of $X$ plus a flat term, or we can write $G=G_{1}(1+$ flat $)$, where $G_{1}$ is a first integral of $X$. Normalizing the new vector field $Y=X / G_{1}$ instead of the old $Y=X / F$, 
we get a new smooth coordinate system in which $P Y=(2 \pi / \lambda) X^{(1)}$, where $P$ is the period function of the new vector field $Y$, and it is a smooth first integral of the type const + flat. In this new coordinate system we have that $X$ is equal to its linear part times a first integral, and Theorem 1.1 is proved in the elliptic case, i.e., without eigenvalue 0 .

Since our proof for the strong hyperbolic case and the strong elliptic case also works for smooth families of integrable vector fields, Theorem 1.1 is proved.

Remark 3.3. According to a theorem of Schwarz [9], the smooth first integral $F$ in the normal form in the elliptic case can also be written as

$$
F=f\left(Q_{1}\left(x_{1}, \ldots, x_{n}\right), \ldots, Q_{m}\left(x_{1}, \ldots, x_{n}\right)\right)
$$

where $Q_{1}\left(x_{1}, \ldots, x_{n}\right), \ldots, Q_{m}\left(x_{1}, \ldots, x_{n}\right)$ are homogeneous polynomials which generate the ring of polynomial first integrals of the linear vector field $X^{(1)}$.

\section{The case of dimension 2}

The aim of this section is to show that condition iii) in Theorem 1.1 is redundant at least in the case of dimension 2. More precisely, we have:

Theorem 4.1. Let $X$ be a smooth vector field in a neighborhood of $O=(0,0)$ in $\mathbb{R}^{2}$, which vanishes at $O$ and satisfies the following conditions:

i) (complete integrability): $X$ admits a smooth first integral $F_{1}$;

ii) (nondegeneracy): the semisimple part of the linear part of $X$ at $O$ is non-zero, and the $\infty$-jet of $F_{1}$ at $O$ is non-constant. Then there exists a local smooth coordinate system $(x, y)$ in which $X$ can be written as

$$
X=F X^{(1)}
$$

where $X^{(1)}$ is a semisimple linear vector field in $(x, y)$, and $F$ is a smooth first integral of $X^{(1)}$.

Proof. Remark that, in the case of dimension 2, there are only 3 possibilities: elliptic without zero eigenvalue, hyperbolic without zero eigenvalue, and hyperbolic with zero eigenvalue. The first two possibilities are covered by Theorem 1.1. It remains to prove Theorem 4.1 for the case when $X$ has one eigenvalue equal to 0 . By Proposition 2.3, we can assume that

$$
X=F(y) x \frac{\partial}{\partial x}+\text { flat }_{1} \frac{\partial}{\partial x}+\text { flat }_{2} \frac{\partial}{\partial y}
$$

in a smooth coordinate system $(x, y)$, where flat and flat $_{2}$ are two flat functions, and $F(0) \neq 0$.

Denote by

$$
S=\{q \in U \mid X(q)=0\}
$$

the singular locus of $X$ near $O$, where $U$ denotes a small neighborhood of $O$ in $\mathbb{R}^{2}$. The main point is to prove that $S$ is a smooth curve. If $S$ is a smooth curve, then we can write $S=\{x=0\}$, the vector field $X$ is divisible by $x$, i.e., $Y=X / x$ is still a smooth vector field, which is non-zero at $O$, and therefore locally rectifiable and admits a first integral $G$ such that $\mathrm{d} G(0) \neq 0$. But $G$ is also a first integral of $X$, so condition iii) of Theorem 1.1 is also satisfied, and Theorem 4.1 is reduced to a particular case of Theorem 1.1. 
Denote by

$$
S_{1}=\left\{(x, y) \in U \mid F(y) x+\operatorname{flat}_{1}(x, y)=0\right\}
$$

the set of points where the $\frac{\partial}{\partial x}$-component of $X$ vanishes. It is clear that $S \subset S_{1}$, and $S_{1}$ is a smooth curve tangent to the line $\{x=0\}$ at $O$ by the inverse function theorem. We will show that $S=S_{1}$.

Consider the open cone

$$
C=\{(x, y) \in U|| x|<| y \mid\} .
$$

Clearly, $S_{1} \subset C \cup\{O\}$ (provided that $U$ is small enough). The non-flat first integral $F_{1}$ of $X$ in the coordinate system $(x, y)$ has the type

$$
F_{1}=f(y)+\text { flat }
$$

where $f(y)=a_{h} y^{h}+$ h.o.t. is a non-flat smooth function. It implies that the level sets of $F_{1}$ in the cone $C$ are smooth curves (because $\frac{\partial F_{1}}{\partial y} \neq 0$ in this cone) which are nearly tangent to the lines $\{y=$ const $\}$ (because $\left|\frac{\partial F_{1}}{\partial x}\right|$ is very small compared to $\left|\frac{\partial F_{1}}{\partial y}\right|$ in the cone). In particular, each level set of $F_{1}$ in $C$ intersects with $S_{1}$ at exactly 1 point. Since $X$ is tangent to these

level sets, and the $\frac{\partial}{\partial x}$-component of $X$ vanishes at the intersection points of these level sets with $S_{1}$, it follows that $X$ itself vanishes at these intersection points. But every point of $S_{1}$ is an intersection point of $S_{1}$ with a level set of $F_{1}$. Thus $X$ vanishes on $S_{1}$, and we have $S=S_{1}$.

Remark 4.2. Two-dimensional elliptic-like vector fields, i.e., those vector fields whose orbits near a singular point are closed, are also called centers in the literature. There is a recent interesting theorem of Maksymenko [7] about the orbital linearization of the center, without the assumption on the existence of a first integral, but with an assumption on the periods of the periodic orbits. Maksymenko's theorem is similar to and a bit stronger than the elliptic case of Theorem 4.1 because his assumptions are weaker, and the conclusions are the same. His proof is also based on the formal normalization and the blowing-up method.

Remark 4.3. Some of the arguments of the proof of Theorem 4.1 are still valid in the $n$ dimensional case where 0 is an eigenvalue with multiplicity $k \geq 1$. In particular, one can still show that, even without condition iii) of Theorem 1.1, the local singular locus of $X$ is still a smooth $k$-dimensional manifold. However, it is more difficult to show that there is still a local regular invariant $(n-k)$-dimensional foliation. If one can show the existence of this regular invariant foliation, then one can drop condition iii) from the statement of Theorem 1.1 because it is a consequence of the first two conditions. Maybe it is possible to use the techniques of Belitskii-Kopanskii [1] together with a kind of desingularization of the first integrals in order to show the existence of an invariant regular foliation, but we don't have a proof so far.

Remark 4.4. As pointed out by a referee of this paper, there is a less elementary but more dynamical proof of Theorem 4.1 which uses a $C^{r}$-central manifold of $X$, where $r$ can be arbitrarily large.

\section{Acknowledgement}

The first version of this manuscript was available since 2012 as an unpublished preprint (see arXiv:1204.5701v1). It was then revised and submitted during the author's stay at the School of Mathematical Sciences, Shanghai Jiao Tong University, as a visiting professor in 2017. He would like to thank Shanghai Jiao Tong University, and especially Tudor Ratiu, Jianshu Li, and Jie $\mathrm{Hu}$ for the invitation, hospitality and excellent working conditions.

The authors would also like to thank the referees of this paper for many pertinent remarks which helped improve the presentation of the paper. 


\section{References}

[1] Belitskii G.R., Kopanskii A.Ya., Equivariant Sternberg-Chen theorem, J. Dynam. Differential Equations 14 (2002), 349-367.

[2] Chaperon M., A forgotten theorem on $\mathbf{Z}^{k} \times \mathbf{R}^{m}$-action germs and related questions, Regul. Chaotic Dyn. 18 (2013), 742-773.

[3] Chen K.-T., Equivalence and decomposition of vector fields about an elementary critical point, Amer. J. Math. 85 (1963), 693-722.

[4] Dufour J.-P., Zung N.T., Poisson structures and their normal forms, Progress in Mathematics, Vol. 242, Birkhäuser Verlag, Basel, 2005.

[5] Golubitsky M., Stewart I., Schaeffer D.G., Singularities and groups in bifurcation theory, Vol. II, Applied Mathematical Sciences, Vol. 69, Springer-Verlag, New York, 1988.

[6] Jiang K., Local normal forms of smooth weakly hyperbolic integrable systems, Regul. Chaotic Dyn. 21 (2016), 18-23.

[7] Maksymenko S.I., Symmetries of center singularities of plane vector fields, Nonlinear Oscil. 13 (2010), 196-227, arXiv:0907.0359.

[8] Malgrange B., Ideals of differentiable functions, Tata Institute of Fundamental Research Studies in Mathematics, Vol. 3, Tata Institute of Fundamental Research, Bombay, Oxford University Press, London, 1967.

[9] Schwarz G.W., Smooth functions invariant under the action of a compact Lie group, Topology 14 (1975), 63-68.

[10] Sternberg S., On the structure of local homeomorphisms of euclidean $n$-space. II, Amer. J. Math. 80 (1958), 623-631.

[11] Ziglin S.L., Bifurcation of solutions and the nonexistence of first integrals in Hamiltonian mechanics. I, Funct. Anal. Appl. 16 (1982), 181-189.

[12] Zung N.T., Convergence versus integrability in Poincaré-Dulac normal form, Math. Res. Lett. 9 (2002), 217-228, math.DS/0105193.

[13] Zung N.T., Non-degenerate singularities of integrable dynamical systems, Ergodic Theory Dynam. Systems 35 (2015), 994-1008, arXiv:1108.3551.

[14] Zung N.T., Geometry of integrable non-Hamiltonian systems, in Geometry and Dynamics of Integrable Systems, Editors E. Miranda, V. Matveev, Advanced Courses in Mathematics, CRM Barcelona, Birkhäuser/Springer, Cham, 2016, 85-140, arXiv:1407.4494.

[15] Zung N.T., Minh N.V., Geometry of integrable dynamical systems on 2-dimensional surfaces, Acta Math. Vietnam. 38 (2013), 79-106, arXiv:1204.1639. 